\title{
SERS of dye film deposited onto gold nano-clusters
}

\author{
K. Grytsenko*, Yu. Kolomzarov, O. Lytvyn, T. Doroshenko, V. Strelchuk \\ V. Lashkaryov Institute of Semiconductor Physics, NAS of Ukraine, \\ 41, prospect Nauky, 03028 Kyiv, Ukraine \\ *Corresponding author: +380 44 525-55-30; e-mail: d.grytsenko@gmail.com
}

\begin{abstract}
Gold nanoclusters were obtained by co-deposition of and polytetrafluoroethylene (PTFE) in vacuum with various gold concentrations. The films deposited were undergone to heating at various temperatures in air. Transformation of ensemble morphology after heating was studied using atomic force microscope (AFM). Raman scattering spectra of an ultra-thin film of Rhodamine 6G deposited onto substrates with gold nano-clusters of different morphology were recorded. The best substrate gave strong amplification of the Raman scattering signal from Rhodamine $6 \mathrm{G}$ film. Therefore, produced $\mathrm{Au}$ nano-clusters are suitable for surface enhanced Raman scattering spectroscopy of nano-quantities of material.
\end{abstract}

Keywords: gold, polytetrafluoroethylene, film, annealing, nano-cluster, dye, SERS.

Manuscript received 28.12.09; accepted for publication 25.03.10; published online 30.04.10.

\section{Introduction}

Recently gold nano-clusters (AuNc) were used for surface enhanced Raman scattering (SERS) of their both organic compounds and inorganic materials. Fields of applications are rather wide: medicine, biology, semiconductors, sensors, research on the processes during optical recording and quality of flat displays [14]. Addition of AuNC to dye layers increases light output from electroluminescent devices [5]. Different wet and gas phase deposition methods are used for AuNc production. They are reviewed in [6] together with one particular method: co-deposition of gold (Au) and polytetrafluoroethylene (PTFE) vapours in vacuum.

The aim of this work is to investigate how the AuNc parameters influence SERS signal from dye ultrathin film.

\section{Experimental details}

Films were deposited using УВН-74 (USSR) installation with a Pfeiffer vacuum pressure meter, Sigma quartz thickness monitor, optical spectrometer StellarNet. Rotating glass discs with attached glass and silica slides were used as substrates. PTFE was evaporated with vapor activation by electron cloud. Heated with electric current molybdenium boat was used for $\mathrm{Au}$ and dye evaporation. Optical properties of the growing film were monitored by optical spectroscopy in situ. Details can be found elsewhere [6-8]. Au concentration in the film was from 5 to $20 \mathrm{vol}$. \%, film thickness was varied from 50 to $100 \mathrm{~nm}$. Heating the films was made in the homemade oven equipped with optical spectrometer Polytec for spectra recording in situ. Film morphology was studied by an atomic force microscope Nanoscope IIIa Dimension $3000^{\mathrm{TM}}$ at room temperature. Rhodamine $6 \mathrm{G}$ (R6G) films were deposited by evaporation in vacuum onto substrates covered with AuNc. The micro-Raman measurements were carried out in backscattering geometry at room temperature using Horiba Jobin Yvon T64000 system, equipped with an Olympus BX41 confocal optical microscope. The $488.0 \mathrm{~nm}$ line of a Spectra Physics Stability 2018-RM Ar+/Kr+ laser was used as an excitation source. The spatial resolution of the confocal arrangement was about $0.4 \mathrm{~mm}$ in the $\mathrm{X}, \mathrm{Y}$ plane. The maximum laser power on the sample did not exceed $1 \mathrm{~mW}$. Raman peak positions were determined with an error less than $0.15 \mathrm{~cm}^{-1}$.

\section{Experimental results and discussion}

As-deposited films contain AuNc in PTFE matrix with sizes dependent on their concentration $[7,8]$. The size of the AuNc was increased from 7 to $50 \mathrm{~nm}$ by annealing 

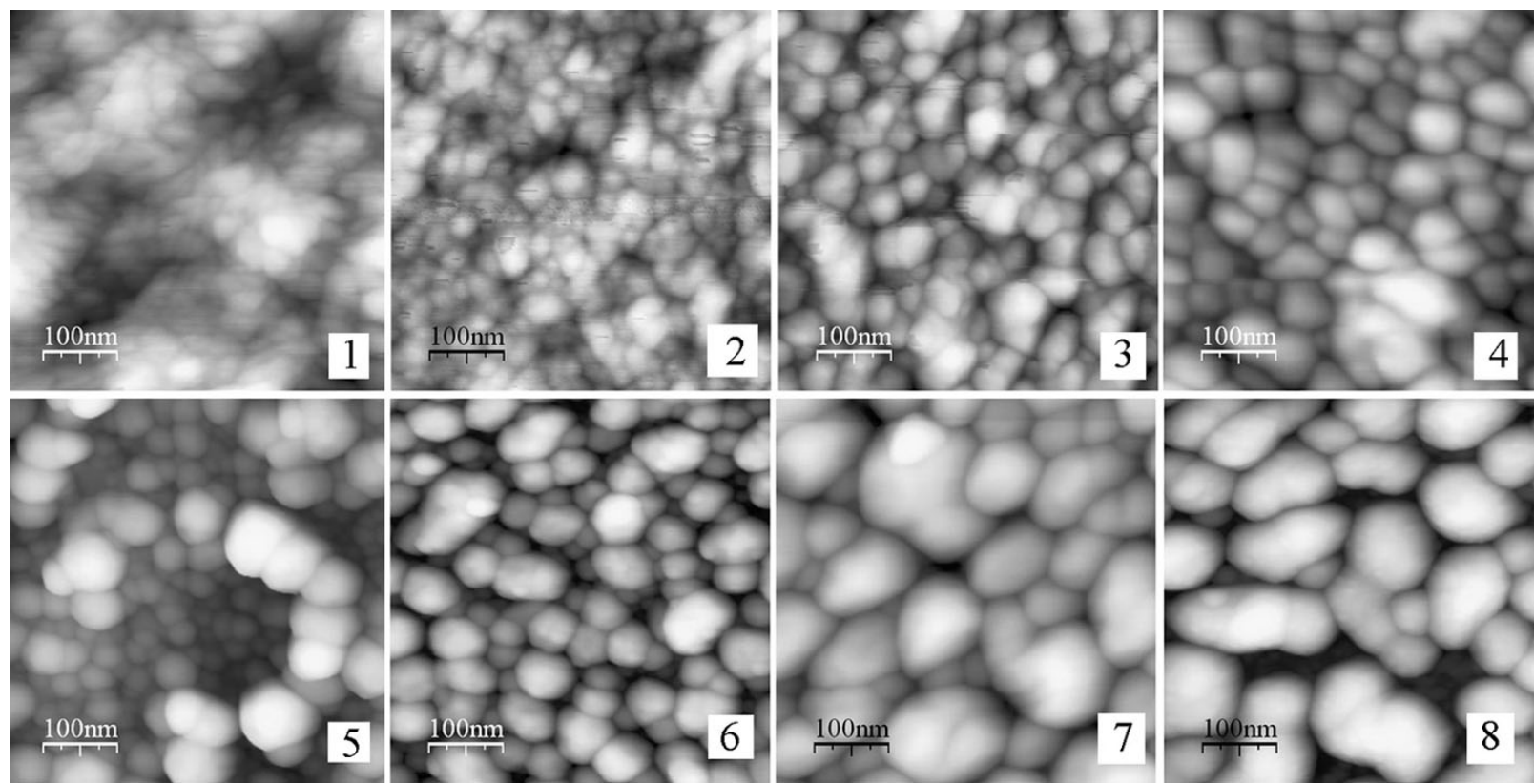

Fig. 1. Morphology of the AuNc films heated up to the temperatures:

$1-150{ }^{\circ} \mathrm{C}, 10$ vol. $\%$ of AuNc; $2-150{ }^{\circ} \mathrm{C}, 15$ vol.\% of AuNc; $3-220{ }^{\circ} \mathrm{C}, 20$ vol. $\%$ of AuNc; $4-220{ }^{\circ} \mathrm{C}, 25$ vol. $\%$ of AuNc; $5-300{ }^{\circ} \mathrm{C}, 10$ vol. $\%$ of AuNc; $6-300{ }^{\circ} \mathrm{C}, 15$ vol.\% of AuNc; $7-300{ }^{\circ} \mathrm{C}, 20$ vol. $\%$ of AuNc; $8-300{ }^{\circ} \mathrm{C}, 25$ vol. $\%$ of AuNc. $\mathrm{Au}$ vol.\% is indicated for as-deposited films.

within the temperature range from 50 to $300 \mathrm{eC}$. Optical absorption spectroscopy in situ following the heating process showed complex shift of the plasmon band maximum wavelength and its shape at various temperatures, in dependence on the AuNc concentration. Several AuNc samples were taken at critical optical points $[9,10]$ for AFM investigation. The surface morphology of the films heated to different temperatures is presented in Fig. 1. The higher were Au concentration and heating temperature, the larger final AuNc mean size. The temperature elevation increased the diffusion rate of Au atoms, PTFE matrix became more soft. The mean size of the AuNc was increased at the first stage of heating by surface diffusion from small clusters to larger ones. At higher temperature, AuNc growth continued, which led to increase of the distance between $\mathrm{AuNc}$ and suppressing the effects related with inter-cluster interactions $[9,10]$.

The SERS of ultra-thin film of R6G (thickness $10 \mathrm{~nm}$ ) deposited onto glass substrate covered with AuNc are presented in Fig. 2. The obtained SERS spectra are in good agreement with the known spectra for R6G presented in the recent publications $[11,12]$. Amplification of the Raman scattering signal is not linearly related with the $\mathrm{Au}$ concentration and heating temperature. The influence of these factors should be determined in details during the next experiments.

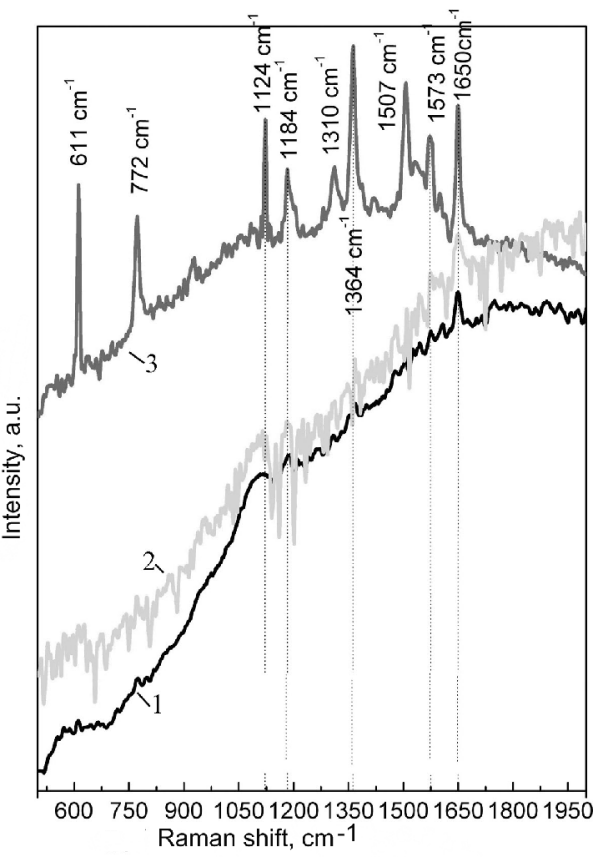

Fig. 2. SERS of the R6G ultra-thin film onto Au nanoclustered surfaces: 1, 2, 3 represent spectra recorded using substrates with different AuNc morphology. 


\section{Conclusions}

Low temperature annealing of gold-PTFE composite thin film allows production of substrate for surface enhanced Raman scattering measurements.

\section{Acknowledgements}

Work was made in the frame of Project No 52 of Ukrainian State Program "Nanostructured systems, nanomaterials, nanotechnologies".

\section{References}

1. P. Podsiadlo, V.A. Sinani, J. Hwan Bahng, N.A. Kotov et al., Gold nanoparticles enhance the antileukemia action of 6-mercaptopurine chemotherapeutic agent // Langmuir, 24, p. 568-574 (2008).

2. A. Convertino, A. Capobianchi, A. Valentini and E.N.M. Cirillo, A new approach to organic solvent detection: High-reflectivity Bragg reflector based on gold nanoparticle/teflon-like composite material // Adv. Materials, 15(13), p. 1103-1105 (2003).

3. Pui-K. Chan and T.R. Hart, Raman scattering temperature probe of laser disk marking // Appl. Opt. 28, p. 1685-1691 (1989).

4. http://www.microspectra.com/support/applicationpapers

5. D.A. Chubich, R.D. Fedorovich, A.G. Vitukhnovsky, Electrical conductivity and luminescence of metal-organic nanocomposite //J. Russian Laser Research 29(4), p. 368-376 (2008).
6. K.P. Gritsenko, A.M. Krasovsky, Thin film deposition of polymers by vacuum degradation // Chem. Rev. 103(9), p. 3607-3650 (2003).

7. K.P. Grytsenko, Growth mechanism, properties and applications of vacuum-deposited PTFE films // Russian J. Chem. Soc. LII(3), p. 112-123 (2008).

8. K. Grytsenko, S. Schrader, Nanoclusters in polymer matrices prepared by co-deposition from a gas phase // Adv. in Colloid and Interface Sci. 116, p. 263-276 (2005).

9. V. Ksenzou, S. Schrader, H. Beyer, K. Grytsenko et al., Control of nanocluster size by annealing or laser treatment of Au-filled PTFE film // Abstr. Book: Conf. "Polymer composites and tribology", Gomel, June 21-25, p. 66 (2009).

10. K.P. Gritsenko, V. Ksensov, S. Schrader, H. Beyer et al., Thin nanocomposite films on PTFE base // Abstr. Book: 8-th Russian Conf. "Fluorine Chemistry-2009“, November 22-25, Chernogolovka, 2009, p. 22.

11. J.A. Diringer, A.D. McFarland, N.C. Shah et al., Surface enhanced Raman spectroscopy: new materials, concepts, characterization tools, applications // Faraday Discuss. p. 1-18 (2005).

12. K. Hering, D. Cialla, K. Ackermann et al., SERS: a versatile tool in chemical and biochemical diagnostics // Anal. Bioanal. Chem. 390, p. 113-124 (2008). 